$H^{*}\left(S^{2 r} \times S^{2 s} ; Z_{p}\right)$ where $2 r$ and $2 s$ are even and positive and $r \leqq m, s \leqq n$.

Furthermore, if $r \neq s$, then $H^{*}\left(X^{\pi} ; Z_{p}\right)$ is isomorphic to

$$
H^{*}\left(S^{2 r} \times S^{2 s} ; Z_{p}\right)
$$

as a ring.

\title{
REFERENCES
}

1. H. Cartan and S. Eilenberg, Homological algebra, Princeton, 1956.

2. E. E. Floyd, On periodic maps and the Euler characteristic of associated spaces, Trans. Amer. Math. Soc. vol. 72 (1952) pp. 138-147.

3. A. Heller, Homological resolutions of complexes with operators, Ann. of Math. vol. 60 (1954) pp. 283-303.

4. P. A. Smith, Fixed points of periodic transformations, Appendix B of Lefschetz, Algebraic topology, Amer. Math. Soc. Colloquium Publications, vol. 27, 1942.

University of Chicago

\section{A COMPLETE CHARACTERIZATION FOR EXTREME FUNCTIONALS}

BY R. C. BUCK ${ }^{1}$

Communicated by A. M. Gleason, February 12, 1959

1. Introduction. Let $E$ be a real linear space with a seminorm \|\| . Let $S$ be the set of linear functionals of norm less than or equal to 1 ; thus, $L \in S$ whenever $|L(x)| \leqq\|x\|$ for all $x \in E$. In many problems, it is important to know something about the extreme points of $S$; $L$ is extreme if we cannot write $L=\left(L^{\prime}+L^{\prime \prime}\right) / 2$ with $L^{\prime}$ and $L^{\prime \prime}$ distinct members of $S$. In this note, a new procedure will be developed for the study of a particular functional $L$; in particular, this will provide a surprisingly simple and useful characterization for the extreme functionals. With each $L$, we shall associate a closed subspace $V_{L}$ of $E$ in such a fashion that the relative "flatness" of $S$ at $L$ is determined by the size of $V_{L}$. In particular, $L$ is an extreme point of $S$ if and only if $V_{L}$ is all of $E$. The results are formulated for a seminormed space $E$ to allow their application to certain special cases of considerable interest. One such question is discussed in the last section where we look for extreme functionals in the class of those that vanish on a fixed subspace $M$.

2. Construction of $V_{L}$. An equivalent way to say that $L$ is extreme in $S$ is to say that a functional $\theta$ obeys $\|L \pm \theta\| \leqq 1$ only if $\theta=0$. Because we are dealing with a real space, this condition on $\theta$ can be rewritten as

${ }^{1}$ John Simon Guggenheim Fellow. 


$$
|\theta(x)| \leqq\|x\|-|L(x)| \quad \text { all } x \in E .
$$

The key to the results in this note is the construction of a special seminorm $p^{*}$ on $E$ which is characterized as being the largest one that is dominated by the right side of (1); we therefore have

$$
p^{*}(x) \leqq\|x\|-|L(x)| \quad \text { all } x \in E .
$$

From the maximality of $p^{*}$, it follows at once that any functional $\theta$ that obeys (1) must also satisfy the condition $|\theta(x)| \leqq p^{*}(x)$ for all $x$. The subspace $V_{L}$ appears as the null space of $p^{*}$; an explicit description of $V_{L}$ is given later. We see at once that if $V_{L}$ is $E$ itself, then $\theta=0$, and $L$ is extreme; on the contrary, if $V_{L}$ (which is necessarily closed since $p^{*}$ by (2) will be continuous) is proper, then the HahnBanach theorem supplies at least one functional $\theta$ obeying $|\theta(x)|$ $\leqq p^{*}(x)$ (and hence (1)) and not identically zero, so that $L$ is not extreme.

It is apparent that we can say more; if $V_{L}$ has finite co-dimension (deficiency) $N$, then there are only $N$ independent functionals $\theta$, and the "face" of $S$ containing the nonextreme point $L$ has dimension $N$ exactly. We have therefore obtained a relatively explicit way to examine the local structure of the unit ball in the dual space of an arbitrary normed linear space.

It is convenient to construct $V_{L}$ and $p^{*}$ together, rather than to use the latter to obtain the former. Once the correct approach has been discovered, the details of the proof involve essentially only routine verifications; accordingly, we omit these and give the definitions and a chain of lemmas which lead to the desired results.

Definition 1. For any integer $k>0$, let $F_{k}$ be the set of $x \in E$ with $\|x\|-L(x) \leqq 1 / k$.

Definition 2. Set $V_{L}=\bigcap_{1}^{\infty}\left(F_{k}-F_{k}\right)$.

LEMmA 1. (i) $F_{1} \supseteq F_{2} \supseteq F_{3} \supseteq \cdots$, (ii) if $\|x\| \leqq 1 / 2 k$, then $x \in F_{k}$, (iii) given $r>0$ and $k$, choose $k^{\prime} \geqq r k$. Then, $r F_{k^{\prime}} \subseteq F_{k}$. (iv) $F_{2 k}+F_{2 k} \subseteq F_{k}$.

From this, we observe that $F_{k}$ is a convex set containing a neighborhood of the origin.

\section{LemMa 2. $V_{L}$ is a subspace of $E$.}

It will appear later that $V_{L}$ is always a closed subspace. Note that a point $y$ belongs to $V_{L}$ if and only if $y$ can be expressed in the form $u-v$ with $u$ and $v$ in $F_{k}$, and this holds for each $k$. Several simple examples will help in visualizing this. Choose $E$ as the plane with the usual norm $\|x\|=\left\{x_{1}^{2}+x_{2}^{2}\right\}^{1 / 2}$ and $L(x)=L\left(x_{1}, x_{2}\right)=x_{1}$. Then, $F_{k}$ is a parabolic region and $V_{L}=E$. If we modify the norm to $\|x\|=\left|x_{1}\right|$ 
$+\left|x_{2}\right|$ then $F_{k}$ becomes an unbounded polygonal region containing the positive horizontal axis, and $F_{k}-F_{k}$ is the strip $\left\|x_{2}\right\| \leqq 2 / k$; accordingly, $V_{L}$ is now the horizontal axis itself.

Lemma 3. If $V_{L}=E$, then $L$ is extreme in $S$.

Introduce $\theta$ as above, obeying (1). Then, if $u \in F_{k},|\theta(u)| \leqq 1 / k$. Thus, if $y \in V_{L}$, then $y=u-v$ with $u$ and $v$ in $F_{k}$, so that $|\theta(y)|$ $\leqq|\theta(u)|+|\theta(v)| \leqq 2 / k$. Hence, $\theta(y)=0$, and $\theta$ must vanish on $V_{L}$; if $V_{L}=E, \theta$ is identically zero, and $L$ is extreme.

Definition 3. $p_{k}(x)=\inf _{u \in F_{k}}\|x+u\|-L(x+u)$.

Definition 4. $p(x)=\lim _{k \rightarrow \infty} p_{k}(x)$.

This limit exists for each $x$, since $0 \leqq p_{1}(x) \leqq p_{2}(x) \leqq p_{k}(x) \leqq\|x\|$ $-L(x)$.

Lemma 4. (i) If $x \in F_{k}-F_{k}$, then $p_{k}(x) \leqq 1 / k$, (ii) if $p_{k}(x)<1 / k$, then $x \in F_{k}-F_{k}$, (iii) for any $x$ and $y$ in $E, p_{k}(x+y) \leqq p_{2 k}(x)+p_{2 k}(y)$, (iv) given $r>0$, and $k$, there exist $k^{\prime}$ and $k^{\prime \prime}$ larger than $k$ with $p_{k^{\prime}}(r x) \leqq r p_{k^{\prime \prime}}(x)$.

Lemma 5. The function $p$ is almost a seminorm on $E$ : (i) for any $x$ and $y, p(x+y) \leqq p(x)+p(y)$, (ii) if $r>0$, then $p(r x)=r p(x)$, (iii) $p(x)$ $=0$ if and only if $x \in V_{L}$, (iv) $V_{L}$ is a closed subspace of $E$.

Definition 5. $p^{*}(x)=\inf _{z \in E} p(x+z)+p(z)$.

Lemma 6. $p^{*}$ is a seminorm on $E$ : (i) for any $x$ and $y, p^{*}(x+y)$ $\leqq p^{*}(x)+p^{*}(y)$, (ii) $p^{*}(r x)=|r| p^{*}(x)$ for all real $r$, (iii) $p^{*}(x) \leqq\|x\|$ $-|L(x)|$ for all $x$, (iv) $p^{*}(x)=0$ if and only if $x \in V_{L}$, (v) $p^{*}$ is the largest seminorm on $E$ obeying (iii).

The argument outlined at the start of this section now establishes the main result.

TheOREM 1. A functional $L$ in $S$ is extreme if and only if every point $x \in E$ can be expressed in the form $u-v$, with $u$ and $v$ in $F_{k}$, for every $k$.

3. Constrained extremals. Let $M$ be a closed subspace of $E$, and denote by $S_{M}$ the functionals $L$ of norm not exceeding 1 which vanish on $M$.

Theorem 2. $L$ in $S_{M}$ is extreme in $S_{M}$ if and only if $\left(F_{k}-F_{k}\right)+M$ $=E$, for each $k$.

This may be deduced directly from Theorem 1 . Introduce the new seminorm $\|x\|^{\prime}=\inf _{m \in M}\|x-m\|$, and observe that for any $L \in S_{M}$, we still have $|L(x)| \leqq\|x\|^{\prime}$ for all $x$. Constructing the associated sets $F_{k}^{\prime}$, where $x \in F_{k}^{\prime}$ if and only if $\|x\|^{\prime}-L(x) \leqq 1 / k$, we apply the main 
theorem to conclude that $L$ is extreme in $S_{M}$ if and only if $F_{k}^{\prime}-F_{k}^{\prime}$ $=E$ for each $k$. Clearly, $F_{k}+M \subseteq F_{k}^{\prime}$, so that $\left(F_{k}-F_{k}\right)+M \subseteq F_{k}^{\prime}-F_{k}^{\prime}$. In the other direction, if $u \in F_{2 k}^{\prime}$, then for some $m \in M$,

$$
\|u-m\|-L(u)<1 / k
$$

so that $u \in F_{k}+M$. Accordingly, $F_{2 k}^{\prime}-F_{2 k}^{\prime} \subseteq\left(F_{k}-F_{k}\right)+M$, and we see that $V_{L}^{\prime}=\bigcap_{1}^{\infty}\left(F_{k}^{\prime}-F_{k}^{\prime}\right)=\bigcap_{1}^{\infty}\left\{\left(F_{k}-F_{k}\right)+M\right\}$, proving Theorem 2 .

It seems probable that $V_{L}+M$ is in fact always dense in $V_{L}^{\prime}$, although I do not have a proof of this at the moment. It would also be of interest to know if there is an analogous result for complex linear spaces.

In a paper to appear later, I will apply these methods to discuss the approximation of functions by solutions of a homogeneous differential equation. One example will show the connection. Choose $E$ as the space of real functions continuous on a closed region $\Omega$, and $M$ as the subspace of those that are harmonic in the interior of $\Omega$. For a function $\phi \in E$, we wish to estimate the optimal error in approximating $\phi$ by harmonic functions; for this, we need to know the extreme linear functionals $L$ of norm 1 among those that vanish on the subspace $M$. In the determination of these, Theorem 3 proves to be a very effective criterion.

STANFORD UNIVERSITY AND

UNIVERSITY OF WISCONSIN 